\title{
Endothelins are vascular-derived axonal guidance cues for developing sympathetic neurons
}

\author{
Takako Makita ${ }^{1}$, Henry M. Sucov ${ }^{2}$, Cheryl E. Gariepy ${ }^{3}$, Masashi Yanagisawa ${ }^{4}$ \& David D. Ginty ${ }^{1}$
}

\begin{abstract}
During development, sympathetic neurons extend axons along a myriad of distinct trajectories, often consisting of arteries, to innervate one of a large variety of distinct final target tissues. Whether or not subsets of neurons within complex sympathetic ganglia are predetermined to innervate select end-organs is unknown. Here we demonstrate in mouse embryos that the endothelin family member Edn3 (ref. 1), acting through the endothelin receptor EdnrA (refs 2,3), directs extension of axons of a subset of sympathetic neurons from the superior cervical ganglion to a preferred intermediate target, the external carotid artery, which serves as the gateway to select targets, including the salivary glands. These findings establish a previously unknown mechanism of axonal pathfinding involving vascular-derived endothelins, and have broad implications for endothelins as general mediators of axonal growth and guidance in the developing nervous system. Moreover, they suggest a model in which newborn sympathetic neurons distinguish and choose between distinct vascular trajectories to innervate their appropriate end organs.
\end{abstract}

The sympathetic division of the autonomic nervous system is composed of preganglionic sympathetic neurons, derived from the neuroectoderm, and their synaptic targets, neural-crest-derived postganglionic sympathetic neurons, the cell bodies of which reside within paravertebral and prevertebral sympathetic ganglia. Postganglionic sympathetic neurons (referred to hereafter as sympathetic neurons) innervate and control a wide range of targets, including exocrine and endocrine glands and ducts, smooth muscle layers of the intestine and blood vessels, cardiac muscle and nodes, and pilomotor muscles. Sympathetic neurons originate during development when neural crest cells bilaterally delaminate from the neural tube, migrate and assemble into ganglia. In mouse embryos, aggregation of neural crest cells into sympathetic chain ganglia initiates at approximately embryonic day $10.5(\sim$ E10.5), adjacent to the dorsal aorta. The cranial-most sympathetic ganglia, the superior cervical ganglia (SCG), ultimately come to reside adjacent to the bifurcation of the internal and external carotid arteries from the common carotid arteries. Sympathetic neurons of the SCG send axonal projections either along the external carotid arteries to innervate the submandibular, sublingual and parotid glands or along the internal carotid arteries to the lacrimal and pineal glands, the eye, blood vessels and skin of the head, and the mucosa of the oral and nasal cavities.

A fundamental, unanswered question is how subsets of neurons within any complex autonomic ganglion, such as the SCG, acquire characteristic molecular and functional properties appropriate for the control of their respective end organs. One model posits that axons emanating from the SCG or other ganglia grow along intermediate targets, such as blood vessels, in a random or stochastic manner, and that it is the end organ that retrogradely specifies morphological and biochemical features, presynaptic connections and physiologic properties of the innervating neuron. Evidence in support of this model derives from the observation that a small subset of sympathetic neurons acquires a cholinergic transmitter phenotype on innervation of eccrine sweat glands and acquisition of a sweatgland-derived cholinergic differentiation factor $(\mathrm{CDF})^{4}$. A second model posits that subpopulations of newly born sympathetic neurons are molecularly distinct; these subpopulations choose from several intermediate target structures, such as particular blood vessels that serve to guide axonal trajectories to their appropriate end organs. Identification of axonal guidance cues that direct select subsets of sympathetic axons towards one or another vascular path would serve as evidence in support of the latter model. We therefore asked whether axons of developing sympathetic neurons of the SCG choose between extending along either the internal or the external carotid arteries because these blood vessels provide the main routes by which SCG axons project to distinct end-organs in the head and neck. These investigations led us to define the molecular identity of the cues and their receptors that guide extending axons along the vascular network.

In mouse embryos, the SCG form in the upper thorax by E11.5 as a condensation of neural precursors dorsal to the outflow tract of the heart. SCG neurons extend axons cranially along the primitive internal carotid arteries (derived from the dorsal aortae; Fig. 1a, d). At this stage, the external carotid arteries are beginning to emerge from the third pharyngeal arch arteries near their origins from the aortic $\mathrm{sac}^{5}$; there are no projections of sympathetic axons along either the nascent external carotid arteries or the third arch arteries (Fig. 1a, g). By E12.5, the common carotid arteries have elongated and the external carotid arteries are substantially developed. At this time, the sympathetic chain has become delimited into distinct ganglia and the SCG are clearly distinct from the more caudal stellate ganglia. Sympathetic neurons initiate extension of axons towards the external carotid arteries at this time, originating from the SCG at the level of the junction of the base of internal and external carotid arteries (Fig. 1b, m, p); the SCG extends cranially along the internal carotid arteries as far as the stapedial arteries (Fig. 1b, j). By E13.5, SCG axonal projections associated with both the external and internal carotid arteries are more substantially developed, although the general pattern of blood vessels and axonal projections is the same as the previous day (Fig. 1c, q, t, w and Supplementary Figs 1 and 2).

Previous studies using Wnt1Cre and R26R mice have defined the neural crest and mesodermal origins of vascular smooth muscle in the cardiac outflow tract and great blood vessels, although not specifically of the vascular segments relevant for SCG projections ${ }^{6,7}$. The

\footnotetext{
The Solomon H. Snyder Department of Neuroscience, Howard Hughes Medical Institute, The Johns Hopkins University School of Medicine, Baltimore, Maryland 21205, USA. ${ }^{2}$ Institute for Genetic Medicine, University of Southern California Keck School of Medicine, Los Angeles, California 90033, USA. ${ }^{3}$ Department of Pediatrics and Communicable Diseases, University of Michigan, Ann Arbor, Michigan 48109, USA. ${ }^{4}$ Department of Molecular Genetics, Howard Hughes Medical Institute, University of Texas Southwestern Medical Center, Dallas, Texas 75235, USA.
} 
third pharyngeal arch arteries are lined by neural-crest-derived smooth muscle (Fig. 1h, i). Consequently, their derivatives, which include the entirety of the external carotid arteries and the base of the internal carotid arteries, also have a neural crest origin (Fig. 1n, o, u, v and Supplementary Fig. 2). In contrast, the dorsal aortae are lined by smooth muscle that is mesodermal in origin (Fig. 1h, i) and, as a result, the internal carotid arteries from just above their origination from the common carotid arteries have a mesoderm-derived smooth muscle layer (Fig. 1e, f, k, l, r, s and Supplementary Fig. 2). Thus, the smooth muscle layers of the internal and external carotid arteries arise from different cell lineages and may therefore also be molecularly distinct. These arteries may, therefore, serve as distinct axonal

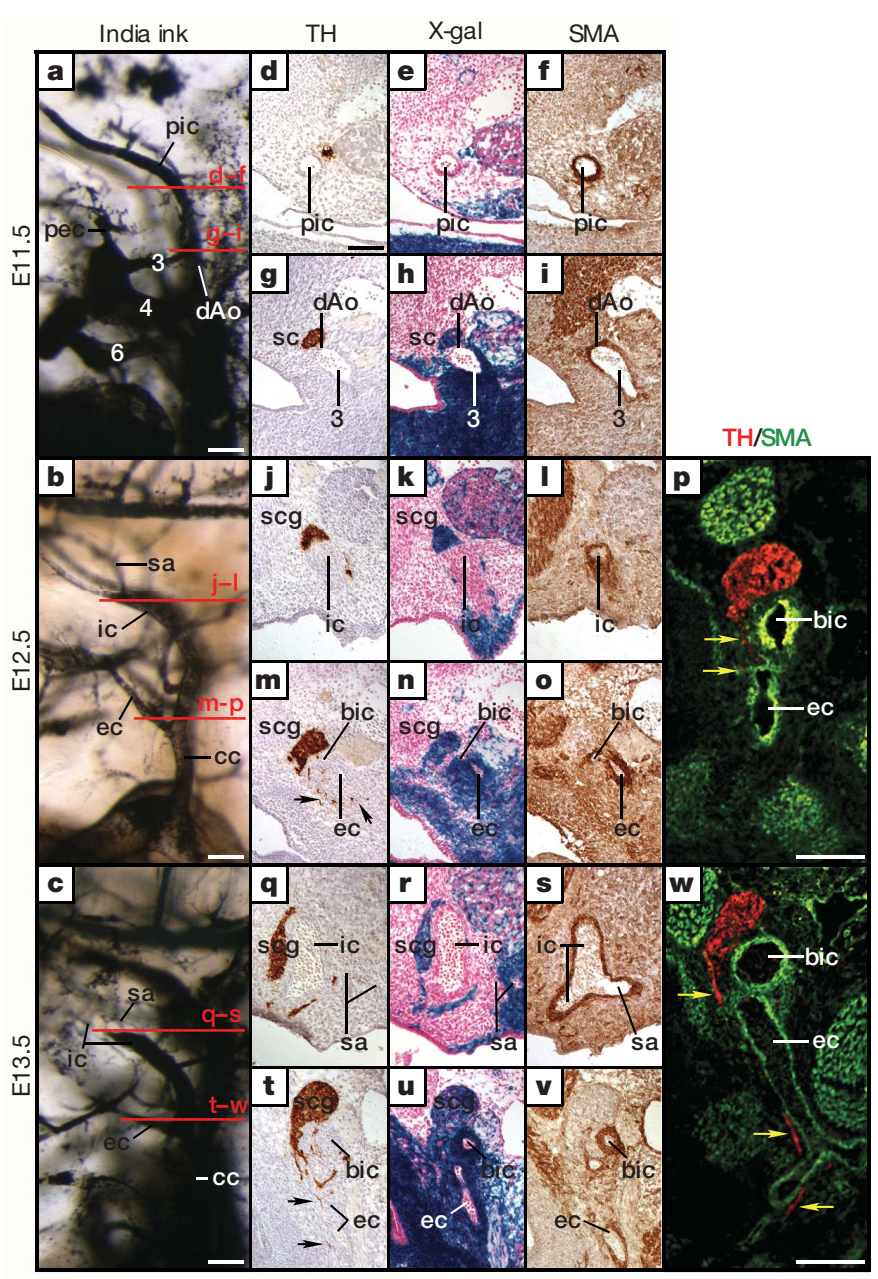

Figure 1 | Development of vascular and sympathetic systems. a-c, India ink visualization of the carotid arteries. The red lines indicate the approximate levels of sections shown in $\mathbf{d}-\mathbf{w}$. $\mathbf{d}-\mathbf{w}$, Serial transverse sections at the approximate levels indicated in panels a-c were stained with X-gal or immunostained for TH or alpha smooth muscle actin. $\mathbf{d}-\mathbf{f}, \mathbf{j}-\mathbf{I}$ and $\mathbf{q}-\mathbf{s}$ are sections through the internal carotid artery. $\mathbf{g}-\mathbf{i}, \mathbf{m}-\mathbf{p}$ and $\mathbf{t}-\mathbf{w}$ are at the level of the carotid bifurcation and show the base of the internal and external carotid arteries. $\mathbf{m}, \mathbf{t}$, Arrows denote SCG axons extending along the external carotid artery. Low-magnification views of the same sections are provided in Supplementary Fig. 1. At least ten embryos at each stage were analysed in this manner with comparable results. $\mathbf{p}, \mathbf{w}$, Confocal immunofluorescent images of sections of E12.5 and E13.5 embryos at a level approximately as indicated in $\mathbf{b}$ and $\mathbf{c}$, respectively, showing TH-positive axons (red) projecting along the smooth muscle layer (green) of the base of internal and external carotid arteries (yellow arrows). Abbreviations: 3,4 and 6, third, fourth and sixth pharyngeal arch arteries, respectively; bic, base of internal carotid artery; cc, common carotid artery; dAo, dorsal aorta; ec, external carotid artery; ic, internal carotid artery; pec, primitive external carotid artery; pic, primitive internal carotid artery; sa, stapedial artery; sc, sympathetic chain; and scg, superior cervical ganglion. Scale bars, $100 \mu \mathrm{m}$. growth environments that preferentially attract one, but not the other, of the two main axon bundles emanating from the SCG.

To address this possibility and to identify previously unknown vascular-derived guidance cues for SCG neurons that are selectively expressed by the crest-derived external carotid and the mesodermderived internal carotid arteries, we isolated these vascular segments from embryos at E13.5 and extracted RNA to screen microarrays for differentially expressed genes. Transcripts for endothelin converting enzyme 1 (ECE1), the protease responsible for conversion of inactive precursors (big endothelins) to bioactive forms of endothelins ${ }^{8,9}$, were identified as being selectively expressed in the developing external carotid arteries.

To confirm this finding, and to address the role of endothelins and their cell surface receptors in SCG axonal guidance, we next examined the expression patterns of endothelin signalling components by in situ hybridization on serial sections from Wnt1Cre/R26R embryos at E11.5-E14.5. X-gal staining was used to identify neuralcrest-derived cells, and alpha-smooth muscle actin (SMA) immunohistochemistry was used to label smooth muscle. We found that Ece1 was selectively expressed in the neural-crest-derived smooth muscle layer of the external carotid arteries, but was absent, or present at much lower levels, in mesoderm-derived smooth muscle of the internal carotid arteries (Fig. 2b, h). Ece1 mRNA was most abundant at E11.5 in the third pharyngeal arch arteries (the source of the external carotid arteries), was somewhat lower at E12.5 in the newly formed external carotid arteries, and was only marginally detectable at E13.5 and E14.5 (data not shown). At all stages examined, ECE1 was preferentially associated with the external carotid arteries relative to the internal carotid arteries. Expression of Ece2 has been described, and is not found in this region until E13.5 (when it begins to be expressed in scattered clusters of neurons in the neural tube, dorsal root ganglia and sympathetic ganglia $\left.{ }^{10}\right)$. Of the three endothelins ${ }^{1}$, endothelin 3 ( $E d n 3$ ) mRNA was found at the highest level, and was preferentially expressed around the third arch arteries at E11.5 and associated with the external carotid arteries at E12.5, although there was a small amount of expression detected around the internal carotid arteries at E11.5 and E12.5 (Fig. 2d, j). Similar to Ece1, $E d n 3$ expression was highest at E11.5, was somewhat diminished at E12.5, and was maintained at a low level at E13.5-E14.5. Edn2 was not appreciably expressed in this region during this developmental period (Fig. 2e, k), and $E d n 1$ was expressed in endothelial cells and some scattered smooth muscle and mesenchymal cells at a relatively low level (Fig. 2f, 1). Quantitative real-time PCR measurements using dissected internal and external carotid arteries confirmed the selective expression of Ece1 and $E d n 3$ associated with the external carotid arteries (Fig. 2m). Both endothelin receptor genes, Ednra and Ednrb, encoding EdnrA and EdnrB, respectively ${ }^{2,3}$, were expressed in subpopulations of SCG neurons (Fig. 2n-v). Ednra expression persists through E12.5, although expression abated by E13.5 (Fig. $2 \mathrm{u}$ ) and was nearly undetectable at E14.5 (data not shown). Expression of $E d n r b$, in contrast, was sustained in a small subset of SCG neurons at E13.5 (Fig. 2v) and E14.5 (data not shown). Thus, although previously not appreciated to function in axonal growth and guidance, the spatial and temporal patterns of expression of endothelins, the ECE1 processing enzyme and the endothelin receptors suggest a role for endothelin signalling in guiding subsets of sympathetic axons towards either the internal or the external carotid arteries.

We next used cultured SCG explants grown in a collagen gel support $^{11}$ to ask whether endothelins have the capacity to influence axonal outgrowth of sympathetic axons in vitro. Indeed, robust extension of neurites from E12.5 SCG explants was observed when any of three endothelins was mixed uniformly into the collagen gel, whereas little or no neurite outgrowth was observed when bovine serum albumin (BSA) was provided. SCG explants obtained from E13.5 embryos were equally responsive to endothelins, but no response was observed in E14.5 SCG (Fig. 3a, Supplementary Fig. 3a). To ask whether endothelins are capable of attracting sympathetic 
axons, we next placed E12.5 SCG explants in proximity to beads soaked with endothelins. Human EDN3 seemed to be most effective in this regard (Fig. 3b, middle, and Supplementary Fig. 3b), although EDN1 and EDN2 also promoted directional outgrowth (data not shown). Moreover, when beads soaked with a high concentration of EDN3 were placed adjacent to SCG explants, random or undirected outgrowth was observed in addition to directional outgrowth (Fig. 3b, right). Thus, axonal outgrowth is a concentration-dependent and possibly gradient-dependent process. To determine which endothelin receptor mediates this response, we treated E12.5-E13.5 SCGs with EDN3 in the presence or absence of endothelin-receptor-selective antagonists. The EdnrA antagonist BQ123 (ref. 12) completely eliminated SCG neurite outgrowth, whereas the EdnrB antagonist BQ788 (ref. 13) was ineffective (Fig. 3c, upper row, and Supplementary Fig. 3c). In contrast, outgrowth from the Xth cranial (CN X/vagal)
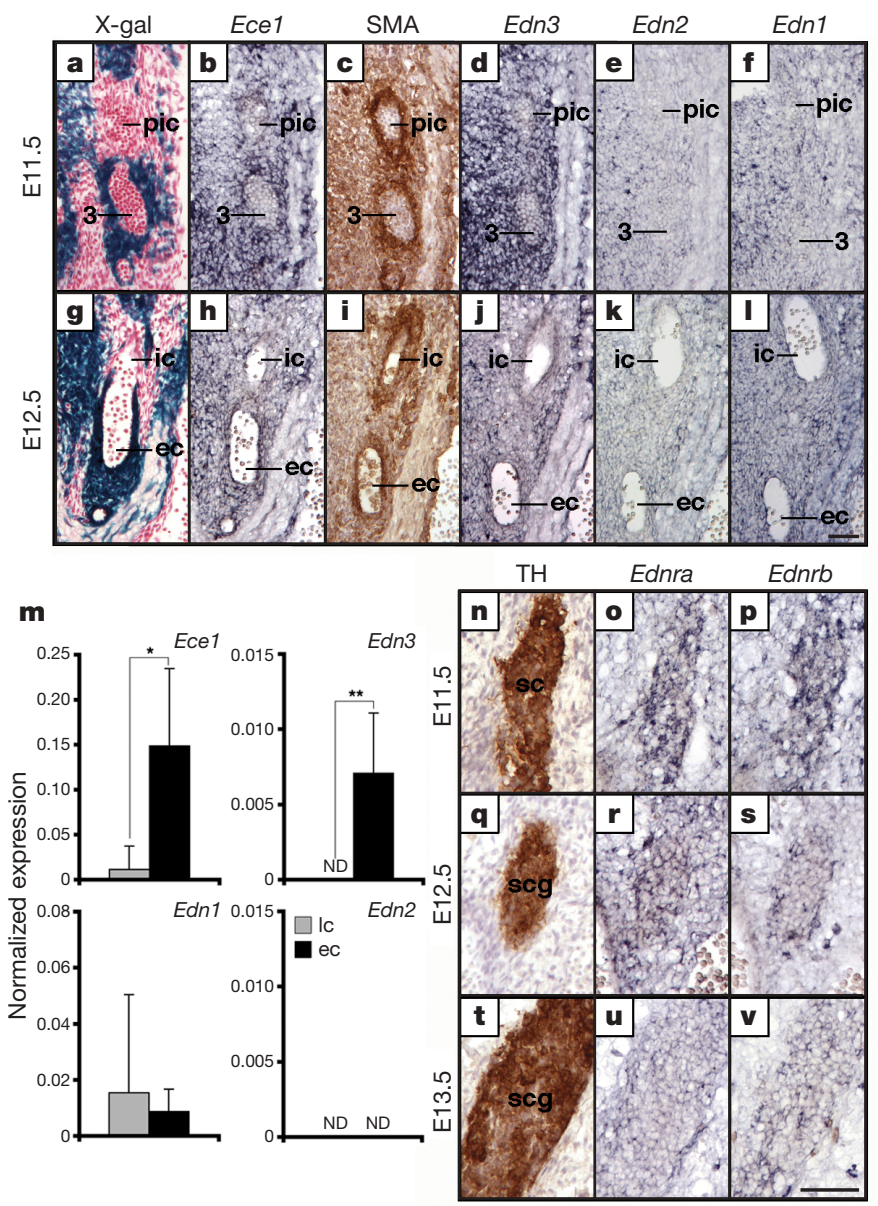

Figure 2 | Expression of endothelin signalling components. a-I, Serial frontal sections of E11.5 (a-f) and E12.5 (g-l) Wnt1Cre/R26R embryos, stained with X-gal, immunostained for SMA, or digoxygenin-labelled by in situ hybridization for the indicated genes. $\mathbf{m}$, Quantitative real-time PCR analysis. Total RNA was extracted from dissected vascular segments from five individual E12.5 wild-type embryos isolated from two different litters. Total RNA was reverse transcribed, and was evaluated for gene expression by quantitative real-time PCR as described in the Methods. Expression levels were normalized to the amount of GAPDH transcripts and are scaled as absolute values. ic and ec, internal and external carotid artery segments. The grey bars represent the signal associated with the internal carotid arteries, whereas the black bars represent the signal associated with the external carotid arteries. ND, not detected. Error bars, mean \pm s.e.m.; ${ }^{*} P<0.01 ;{ }^{* *} P<0.005$. $\mathbf{n}-\mathbf{v}$, Serial frontal sections of embryos at the indicated stages immunolabelled for $\mathrm{TH}$ or labelled by in situ hybridization for Ednra and Ednrb. Sections shown at E11.5 are through the rostral end of the sympathetic chain, and at E12.5 and E13.5 are through the SCG. Results were confirmed with sections from at least five embryos of each stage. Scale bars, $100 \mu \mathrm{m}$. ganglia explants, which express both endothelin receptors (data not shown), was selectively blocked by the EdnrB antagonist (Fig. 3c, lower row, and Supplementary Fig. 3d). We conclude that EDN3-EdnrA signalling is a potent mediator of SCG neurite outgrowth in vitro.

We next asked whether endothelins govern axonal guidance decisions of sympathetic neurons as they encounter the choice of projecting to either the internal or the external carotid arteries in vivo. For these experiments, SCG axonal projections in mice harbouring null mutations in genes encoding endothelin signalling components were assessed. Although EDN2 can promote axonal extension in vitro, $E d n 2$ is not expressed in vascular smooth muscle, and Edn2-null mouse embryos exhibited normal SCG axonal growth along both the internal and the external carotid arteries (data not shown). Similarly, spotting lethal mutant rat embryos, which carry a loss-offunction mutation in the $E d n r b$ gene ${ }^{14}$, also displayed normal SCG axonal projections (Supplementary Fig. 4). Thus, consistent with our

a
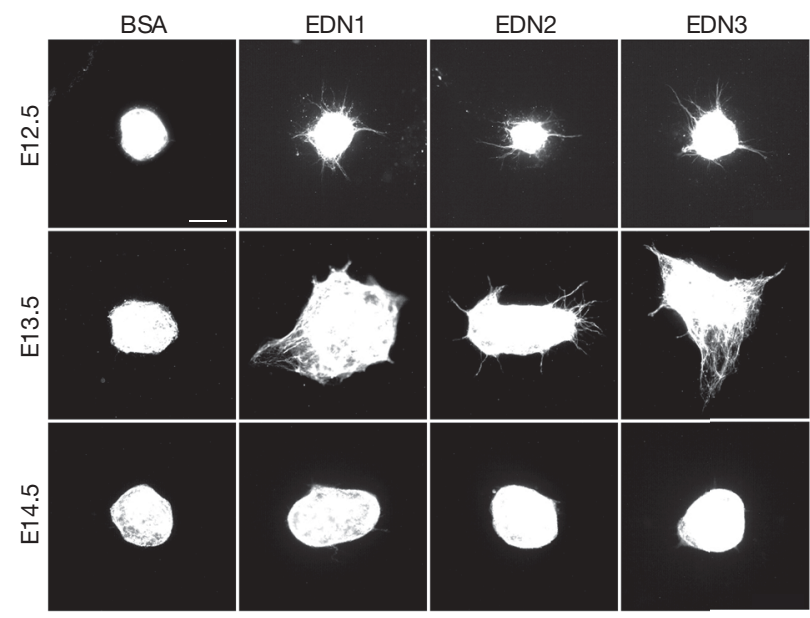

b
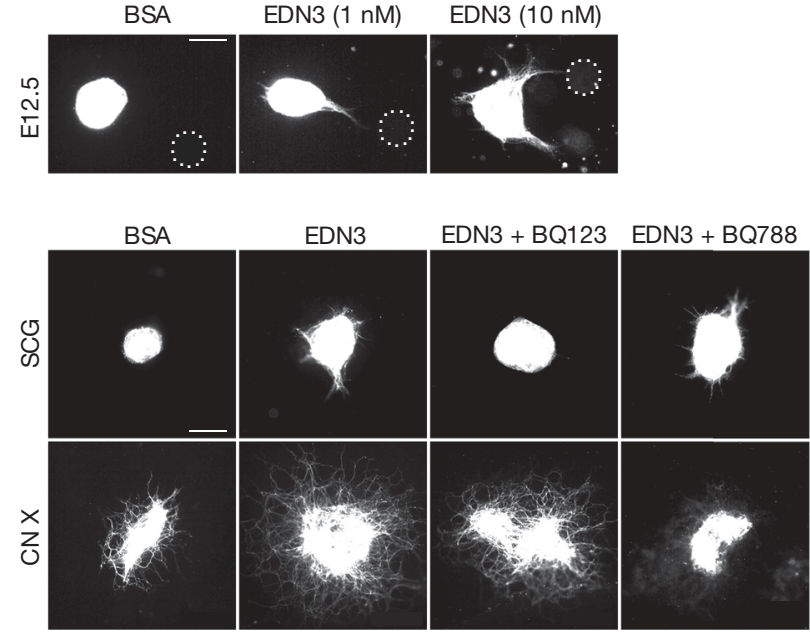

Figure 3 Endothelins promote SCG neurite outgrowth in vitro. a, SCGs from embryos at the indicated stages were harvested and grown in collagen gels prepared with the indicated endothelins $(10 \mathrm{nM})$ or BSA. The average amount of neurite outgrowth from seven separate experiments is shown in Supplementary Fig. 3a. b, Heparin-agarose beads soaked in the indicated concentration of EDN3 or BSA were placed adjacent to E12.5 SCG. Dotted circles indicate the location of the beads. The extent of directional outgrowth was assessed by the relative amount of outgrowth in the proximal direction relative to the distal direction of the beads, as shown in Supplementary Fig. 3b. c, E12.5 SCG (top row) or vagal (bottom row) explants were grown in collagen gels in the presence of EDN3 $(5 \mathrm{nM})$ and the EdnrA antagonist BQ123 $(1 \mu \mathrm{M})$ or the EdnrB antagonist BQ788 $(100 \mathrm{nM})$ as indicated. Quantification of EDN3-induced neurite outgrowth influenced by each antagonist is shown in Supplementary Fig. 3c, d. Scale bars, $100 \mu \mathrm{m}$. 
in vitro results, EdnrB is not required for directed SCG axonal extension. Ece 2 mutant embryos ${ }^{10}$ also exhibited normal SCG axonal outgrowth (data not shown). In contrast, Ednra mutant embryos ${ }^{15}$ exhibited a complete lack of SCG axonal extension along the external carotid arteries (Fig. 4c, i). This axonal projection deficit is specific for the external carotid arteries; Ednra mutants exhibited normal SCG axonal projections along the internal carotid arteries (Fig. 4a, b, g, h). The axonal projection defect in Ednra mutants is not due to defective smooth muscle cell differentiation, as shown by SMA staining (Fig. 4d-f, $\mathrm{j}-\mathrm{l}$ ). Edn3 serves as a ligand that promotes EdnrA signalling in this context because $E d n 3$ mutant embryos ${ }^{16}$ showed a similar although slightly less severe phenotype compared to that of the Ednra mutants, in which SCG axons failed to fully extend along the external carotid arteries (Fig. 4o, r). In Edn3 mutants, SCG neurons did extend some processes around the carotid bifurcation, albeit to a much lesser extent than littermate controls, and they initiated a small and poorly developed projection along the proximal section of the external carotid arteries. Even at E15.5, the latest time point analysed, axonal projections from the SCG along the external carotid arteries and their branches were markedly deficient in $E d n 3^{-1-}$ embryos (Supplementary Fig. 5). We note that Edn1 is also expressed in the vicinity of the developing SCG, and thus Edn1 may have some functional overlap with Edn3 to support modest SCG extension along the external carotid arteries. Moreover, as observed for the Ednra mutants, Edn3-null axons extended along the internal carotid arteries and their branches normally (Fig. 4m, n, p, q and Supplementary Fig. 5).

These in vivo and in vitro findings collectively implicate Edn3 as a vascular-derived sympathetic axon growth factor. We suggest that Edn3, released from the neural-crest-derived smooth muscle layer of the external carotid arteries, directs those SCG neurons destined to innervate the salivary glands to send axonal projections along the nascent external carotid arteries - the sole pathway to these end organs.
Interestingly, our analysis demonstrates that Edn3-EdnrA signalling is not required for projections to the internal carotid arteries. Because embryonic SCGs do not spontaneously exhibit axonal outgrowth, at least in vitro, we predict that other factors expressed by the mesodermderived smooth muscle layer of the internal carotid artery direct projections from the SCG towards this blood vessel. It is noteworthy that the smooth muscle layer of the external carotid arteries originates from the third pharyngeal arch neural crest. Moreover, endothelin signalling also contributes to development of additional third pharyngeal arch structures and other aspects of cardiovascular and craniofacial development ${ }^{15}$, all of which are also dependent on neural crest cell progeny. Furthermore, endothelin receptors are broadly and dynamically expressed in these and other neural crest derivatives. Thus, it will be of interest to ask whether endothelins serve as axonal growth and guidance cues for other neural projections associated with third pharyngeal arch structures and other neural crest derivatives, such as the thymus, thyroid, cardiac outflow tracts and various craniofacial structures. Likewise, endothelins are expressed in the brain and in a variety of mesoderm-derived tissues ${ }^{17}$ and, therefore, may serve as guidance cues for many distinct populations of developing neurons.

Our observations indicate that Ednra expression within the SCG is variegated, suggesting that only a subset of SCG neurons can respond to endothelins and project to the external carotid arteries. The basis of this endothelin receptor heterogeneity within the SCG is unknown; it may arise by stochastic events, by a form of lateral inhibition within the ganglion, or by predetermination within the neural crest sublineage that gives rise to SCG neurons. We do not yet know if EdnrA-positive neurons are also able to project to the internal carotid arteries although, clearly, EdnrA-negative neurons are unable to project to the external carotid arteries. Therefore, we suggest a model in which developing sympathetic neurons are predetermined to choose between distinct vascular trajectories, and are

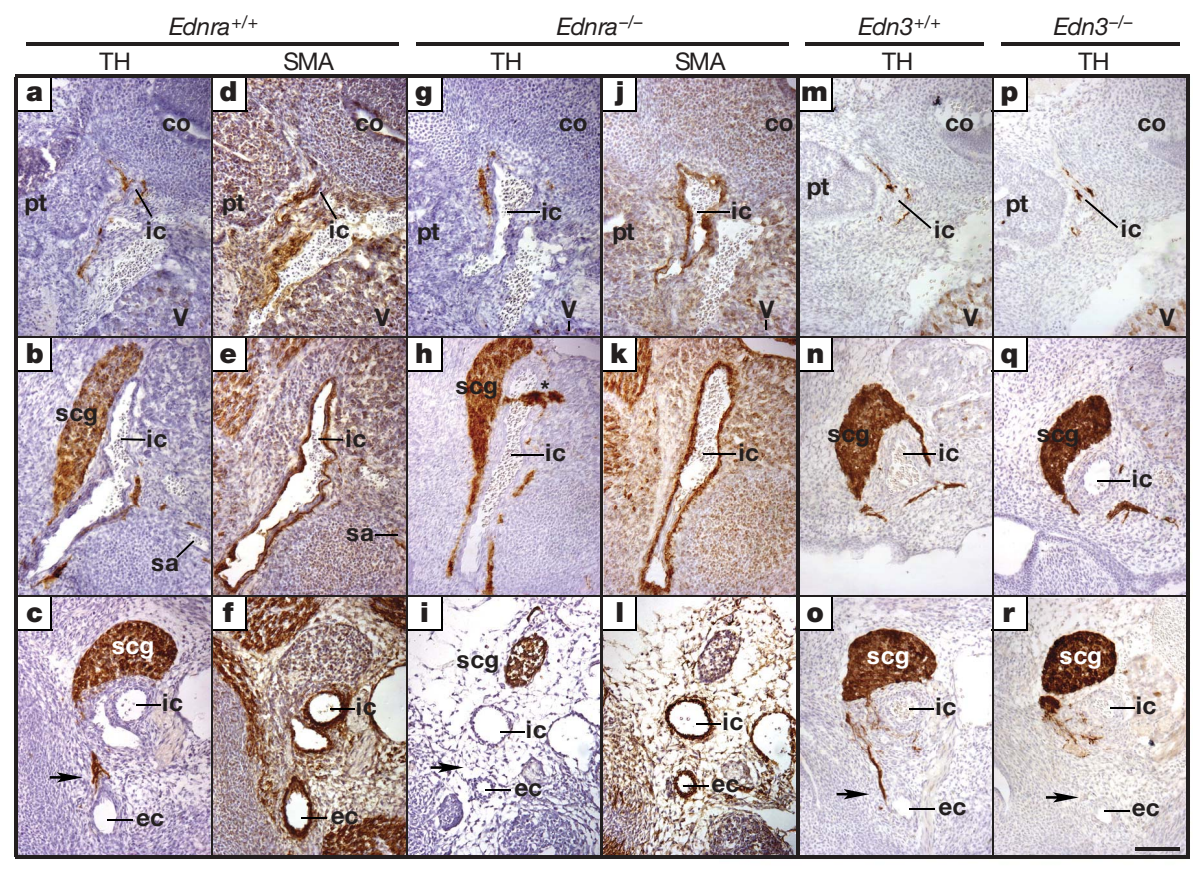

Figure 4 | Edn3 and its receptor EdnrA are essential for SCG axonal projections along the external but not internal carotid arteries in mouse embryos. a-I, Histological sections stained with TH or SMA from an E13.5 $E_{d n r a}{ }^{-1-}$ embryo and a littermate control. Three mutant embryos analysed from multiple litters exhibited a complete absence of axons along the external carotid artery, similar to the embryo shown in $\mathbf{g}-\mathbf{I}$. $\mathbf{m}-\mathbf{r}$, Histological sections from an E13.5 Edn3 ${ }^{-1-}$ embryo and a littermate control stained with TH. Seven $E d n 3^{-1-}$ embryos analysed at E13.5 or older from multiple litters had defects in axonal projections towards the external carotid arteries. Sections shown in the top row are all at the cochlea-pituitary level, and show the rostral projection from the SCG along the internal carotid artery. Shown in the middle row are sections at the level of the branch of the stapedial artery from the internal carotid artery; these reveal the cranial-most extent of the SCG and the initial projection from the SCG to the internal carotid artery. Sections in the lower row were taken at a level immediately above the bifurcation of the external and internal carotid arteries from the common carotid artery. Arrows point to the presence (in panels $\mathbf{c}$ and $\mathbf{o}$ ) or the absence (in $\mathbf{i}$ and $\mathbf{r}$ ) of axonal projections to the external carotid artery. Abbreviations: co, cochlea; $\mathrm{pt}$, pituitary gland; $\mathrm{V}$, trigeminal ganglion; *, histological artefact. Scale bar, $100 \mu \mathrm{m}$. 
thus destined to innervate select end organs. This choice of vascular route is guided, at least in part, by the actions of vascular-derived endothelins.

\section{METHODS SUMMARY}

The Wnt1cre ${ }^{18}, R_{26 R^{19}}, E_{n d n a^{15}}$, spotting lethal ${ }^{14}, E d n 3$ (ref. 16) and Ece2 (ref. 10) alleles have been described previously. X-gal staining and SMA immunostaining of frozen sections were as described previously ${ }^{6,7}$. Tyrosine hydroxylase $(\mathrm{TH}$; Chemicon) immunoreactivity was visualized using an horseradish peroxidase (HRP)-conjugated secondary antibody and a diaminobenzidine (DAB) detection system. For in situ hybridization reactions, digoxygenin (DIG)-labelled antisense probes were generated for each gene, and the hybridization signal was detected using an alkaline phosphatase (AP)-conjugated anti-DIG antibody and 5-bromo-4-chloro-indolylphosphate/nitroblue tetrazolium (BCIP/NTB; Roche). For explant cultures, SCGs were cut into pieces on dissection, and placed into a collagen gel ${ }^{11}$. Explants were grown in the absence or presence of endothelins (Alexis), endothelin- or BSA-soaked heparin-agarose beads (Sigma), or the endothelin receptor antagonists BQ123 or BQ788 (AG Scientific) in Leibovitz$\mathrm{L} 15(\mathrm{~L} 15)-\mathrm{CO}_{2}$ medium supplemented with $5 \%$ fetal bovine serum (FBS) for three days.

Full Methods and any associated references are available in the online version of the paper at www.nature.com/nature.

\section{Received 27 November 2007; accepted 8 February 2008.}

1. Inoue, A. et al. The human endothelin family: three structurally and pharmacologically distinct isopeptides predicted by three separate genes. Proc. Natl Acad. Sci. USA 86, 2863-2867 (1989).

2. Arai, H., Hori, S., Aramori, I., Ohkubo, H. \& Nakanishi, S. Cloning and expression of a cDNA encoding an endothelin receptor. Nature 348, 730-732 (1990).

3. Sakurai, T. et al. Cloning of a cDNA encoding a non-isopeptide-selective subtype of the endothelin receptor. Nature 348, 732-735 (1990).

4. Schotzinger, R. J. \& Landis, S. C. Cholinergic phenotype developed by noradrenergic sympathetic neurons after innervation of a novel cholinergic target in vivo. Nature 335, 637-639 (1988).

5. Hiruma, T., Nakajima, Y. \& Nakamura, H. Development of pharyngeal arch arteries in early mouse embryo. J. Anat. 201, 15-29 (2002).

6. Jiang, X., Rowitch, D. H., Soriano, P., McMahon, A. P. \& Sucov, H. M. Fate of the mammalian cardiac neural crest. Development 127, 1607-1616 (2000).

7. Choudhary, B. et al. Cardiovascular malformations with normal smooth muscle differentiation in neural crest-specific type II TGF $\beta$ receptor (Tgfbr2) mutant mice. Dev. Biol. 289, 420-429 (2006)
8. $\mathrm{Xu}$, D. et al. ECE-1: a membrane-bound metalloprotease that catalyzes the proteolytic activation of big endothelin-1. Cell 78, 473-485 (1994).

9. Yanagisawa, H. et al. Dual genetic pathways of endothelin-mediated intercellular signaling revealed by targeted disruption of endothelin converting enzyme-1 gene. Development 125, 825-836 (1998).

10. Yanagisawa, H. et al. Disruption of ECE-1 and ECE-2 reveals a role for endothelinconverting enzyme-2 in murine cardiac development. J. Clin. Invest. 105, 1373-1382 (2000).

11. Guthrie, S. \& Lumsden, A. Collagen gel coculture of neural tissue. Neuroprotocols 4, 116-120 (1994).

12. Ihara, M. et al. In vitro biological profile of a highly potent novel endothelin (ET) antagonist BQ-123 selective for the ETA receptor. J. Cardiovasc. Pharmacol. 20 (suppl. 12), S11-S14 (1992).

13. Ishikawa, K. et al. Biochemical and pharmacological profile of a potent and selective endothelin B-receptor antagonist, BQ-788. Proc. Natl Acad. Sci. USA 91, 4892-4896 (1994).

14. Gariepy, C. E., Cass, D. T. \& Yanagisawa, M. Null mutation of endothelin receptor type $B$ gene in spotting lethal rats causes aganglionic megacolon and white coat color. Proc. Natl Acad. Sci. USA 93, 867-872 (1996).

15. Clouthier, D. E. et al. Cranial and cardiac neural crest defects in endothelin-A receptor-deficient mice. Development 125, 813-824 (1998).

16. Baynash, A. G. et al. Interaction of endothelin-3 with endothelin-B receptor is essential for development of epidermal melanocytes and enteric neurons. Cell 79, 1277-1285 (1994)

17. Firth, J. D. \& Ratcliffe, P. J. Organ distribution of the three rat endothelin messenger RNAs and the effects of ischemia on renal gene expression. J. Clin. Invest. 90, 1023-1031 (1992).

18. Danielian, P. S., Muccino, D., Rowitch, D. H., Michael, S. K. \& McMahon, A. P. Modification of gene activity in mouse embryos in utero by a tamoxifen-inducible form of Cre recombinase. Curr. Biol. 8, 1323-1326 (1998).

19. Soriano, P. Generalized lacZ expression with the ROSA26 Cre reporter strain. Nature Genet. 21, 70-71 (1999).

Supplementary Information is linked to the online version of the paper at www.nature.com/nature.

Acknowledgements We thank C. Deppmann, A. Kolodkin, J. Merte, S. Sockanathan and L. Schramm for comments on this manuscript, and S. Dixon and N. Murakami for technical support. This research was supported by grants from the National Institutes of Health (to H.M.S., C.E.G., M.Y. and D.D.G.). M.Y. and D.D.G. are investigators of the Howard Hughes Medical Institute.

Author Information Microarray data referred to in this study have been deposited in the Gene Expression Omnibus (GEO, http://www.ncbi.nlm.nih.gov/geo) under the accession number GSE10360. Reprints and permissions information is available at www.nature.com/reprints. Correspondence and requests for materials should be addressed to D.D.G. (dginty@jhmi.edu). 


\section{METHODS}

Histology and immunohistochemistry. Glutaraldehyde-fixed embryos were cryopreserved in sucrose. Five- or ten-micrometre cryosections were post-fixed, stained with X-gal by standard procedures, and counterstained with nuclear fast red. Glutaraldehyde- or paraformaldehyde-fixed embryos were cryopreserved and sectioned at 5- or $10-\mu \mathrm{m}$ thickness for immunostaining. The primary antibodies used were TH (1:400; Chemicon) or alpha-smooth muscle actin (1:1,000; Sigma), and visualized using an HRP-conjugated secondary antibody and $\mathrm{DAB} / \mathrm{H}_{2} \mathrm{O}_{2}$ detection system. For histological examination of sympathetic axonal phenotypes in mutant embryos, all cervical-thoracic sections were immunostained with TH. Alternatively, alternate sections from cervical to thoracic levels were subjected to hematoxylin-eosin staining and TH and SMA immunohistochemistry.

Microarray analysis. Vascular segments (the internal and external carotid arteries) were visualized by India ink injection into the left ventricle and dissected from 22 E13.5 mouse embryos, pooled, and total RNA extracted. Complementary RNAs, synthesized from complementary DNAs made from two rounds of amplification of each RNA sample, was used to hybridize and screen Affymetrix microarrays (MOE430_2) for differentially expressed genes by the JHMI Microarray Core Facility.

In situ hybridization. Histological sections from glutaraldehyde-fixed Wnt1Cre/R26R embryos were used for in situ hybridization reactions. Digoxygeninlabelled sense and antisense probes were generated for Ecel (corresponding to nucleotides 2308-2532 of NM_199307), Edn1 (834-988 of NM_010104), Edn2 (747-851 of NM_007902), Edn3 (1492-1713 of NM_007903), Ednra (565-991 of NM_010332) and Ednrb (1250-1563 of NM_007904), and the hybridization signal was detected using an alkaline-phosphatase-conjugated anti-digoxygenin antibody and BCIP/NTB (Roche).

Collagen gel culture. Vascular structures in E12.5 and E13.5 embryos were visualized by injection of India ink, and SCGs and CN Xs were dissected in PBS. SCGs were cut into several pieces and placed into a collagen gel as described previously ${ }^{11}$. Explants were grown in the absence or presence of synthetic endothelins (EDN1, EDN2 or EDN3, Alexis), the endothelin receptor selective antagonists BQ123 or BQ788 (AG Scientific), or endothelin- or BSA-soaked heparin-agarose beads (Sigma) in $\mathrm{L}_{15 \mathrm{CO}_{2}}$ medium supplemented with $5 \%$ FBS for three days. Synthetic endothelins and receptor antagonists were applied at concentrations ranging from $1 \mathrm{nM}$ to $100 \mathrm{nM}$ and from $10 \mathrm{nM}$ to $10 \mu \mathrm{M}$, respectively, to optimize assay conditions before performing the experiments. Neurite outgrowth was evaluated by immunostaining explants with antineurofilament (2H3) (1:150, Developmental Studies Hybridoma Bank) followed by immunofluorescence detection using fluorescein-conjugated secondary antibodies. To quantify neurite outgrowth, the length of neurites extending from explants was measured using an Image J image processing program.

Quantitative real-time PCR analysis. Vascular segments (the internal and external carotid arteries) were visualized by injection of India ink into the left ventricle and dissected from E12.5 mouse embryos. Total RNA was extracted from individual vascular segment by guanidinium isothiocyanate extraction and were reverse transcribed using standard conditions. All samples were analysed independently by real-time PCR using an iCycler iQ with SYBR green supermix (Bio-Rad). 